\title{
Twisted by DNA
}

\author{
The optical properties of self-assembled plasmonic nanoparticles can reversibly \\ be tuned by using DNA strands.
}

\section{Andrea Di Falco}

Noble-metal nanoparticles (NPs) occupy the top drawer in the toolbox of scientists working with optical materials. These NPs confine light in extremely small volumes, and their individual scattering properties can be designed virtually at will by shaping and arranging the NPs carefully. When their behaviour is considered collectively, the NPs can also be used as building blocks (meta-atoms or metamolecules) for metamaterials - engineered materials with effectively homogeneous properties. In fact, the versatility of metamaterials is to a large degree determined by our ability to shape the units they are made from, and thus their collective properties. In particular, bottom-up fabrication methods based on DNA-assisted NP self-assembly enable the precise arrangement of large volumes of complex metallic assemblies (metamolecules) in three dimensions. Additionally, NPs can be used to transduce topological or physiological information of their surroundings to the optical domain. Actually, the prefix meta-, from the Greek 'beyond', highlights the fact that optical metamaterials permit extremely accurate light control, beyond what is possible with natural materials ${ }^{1,2}$. Indeed, by combining the optical transducing ability of metal NPs with the capability of DNA to execute a programmed design recipe, one can make sensors and media with controlled response to light polarization ${ }^{3,4}$. Now, writing in Nature Materials, Anton Kuzyk, Na Liu and colleagues demonstrate a protocol to tune, dynamically and reversibly, the optical properties of chiral metamolecules by using DNA strands to reconfigure their shape ${ }^{5}$.

Chiral optical media have asymmetric molecules that absorb circularly polarized light, with different absorption efficiency depending on its handedness. Modifying the geometrical configuration of the molecules has an effect on the differential absorption (also called circular dichroism; CD). Kuzyk, Liu and colleagues created chiral metamolecules by placing two gold nanorods on opposite sides of two DNAorigami bundles joined at their centre with a tunable angle. DNA origami involves the folding of a long piece of single-stranded DNA into an arbitrary shape by using DNA staple strands. The DNA staples can be designed computationally to seam together specific sections of the long DNA strand, so that no other assembly configurations are allowed. This greatly simplifies the fabrication procedure because, to create the desired DNA bundles, the basic ingredients can be put all together in a reaction chamber, eliminating the need to execute controlled serial assembly steps. Additionally, the DNA bundles can be designed to offer available binding links for a particular host - the metallic NPs in this case, which are coated with complementary DNA strands. To reversibly control the shape of the DNA-NPs system, and consequently its optical properties, Kuzyk and co-authors decorated the ends of the DNA bundles with two tailored DNA links (Fig. 1). In the neutral, achiral state, the DNA links were capped by matching pieces of DNA (padlocks). By adding specific strands to remove the caps (keys), the authors were able to trigger the locking up of the strands from two nearby ends of the bundles, thus twisting them around their centres and breaking the symmetry of the metamolecules, which acquire chirality. The process is reversible, so that the formed links can be broken and their extremities capped again by introducing nucleotide sequences that match the DNA links.

Kuzyk and co-authors measured the chirality and resulting CD of the metamolecules in solution by comparing the absorption spectra in the various configurations. The authors suggest that the strong CD effect observed could be useful for sensing applications; for example, to monitor the presence of target DNA analytes that can change the chirality of suitably designed molecules. Also, with the authors' method one could change the effective permittivity of solutions of metamolecules, for instance by switching their effective refractive index from positive to negative.

These prospects are exciting, and it is interesting to consider what factors need to be addressed to promote the use of Kuzyk and colleagues' approach outside the controlled laboratory environment. One main issue could be that, despite its intrinsic robustness, the DNA-origami technique is affected by unwanted strand combinations, which reduce the yield of the process and increase the number of required purification steps. This undesired binding events occur at each reconfiguration cycle, and could put a cap on the number of cycles that the molecules can undergo before saturating the dynamics However, these limitations could be overcome with improvements in DNA engineering. 
Another potential limitation in DNA-mediated optical-tuning applications is the speed at which the DNA dynamics can be driven. For example, although absolute speed is not a relevant figure of merit in the work of Kuzyk and collaborators, it is instructive to observe that complete switching times are in the range of minutes, mostly driven by a diffusive dynamics. Although it is clear that this technology is not intended to replace ultrafast optoelectronics devices, one could imagine methods to accelerate the tuning dynamics, for instance by using microfluidics to pump DNA strands in the reaction chamber rather than relying on diffusion alone. An alternative possibility would be to trigger the DNA-tuning mechanism electrically or optically, as has been achieved in supramolecular DNA assembly and DNA machines ${ }^{7}$.

Kuzyk and co-authors' approach could also be extended to different configurations. The authors point out that metamolecules in solution naturally provide an effective isotropic response because the optical properties of the solution are determined by the average of all possible orientations of the metamolecules. This is desirable for properties like chirality or for the definition of a material with a tailored effective dispersion, but it is a limiting factor for applications that would suffer from a dilution or averaging of the optical response, like surface enhanced Raman spectroscopy (SERS) and single-molecule detection and analysis. In particular, SERS relies on high electric-field enhancement between closely packed plasmonic particles, but to obtain repeatable and quantitative SERS analysis it is best to use regular arrangements of the plasmonic features ${ }^{8}$, or to use the response of single isolated nanostructures ${ }^{3}$. An interesting way forward would be a hybrid approach where the metamolecules are arranged on a patterned substrate and driven by DNA assembly.

If DNA-based techniques can be efficiently scaled up to create larger and larger assemblies, an exciting possibility would be to use statistical and nonlinear-dynamics approaches to tailor the collective behaviour of large ensembles of simple components by controlling their mutual interaction ${ }^{9}$. Such complex systems could be used to design biointegrated assemblies that respond strongly to small external control stimuli and whose behaviour can be made to self-evolve following the recipes embedded in the DNA sequence.

Andrea Di Falco is at the School of Physics and Astronomy at the University of St Andrews, St Andrews, United Kingdom.

e-mail:adf10@st-andrews.ac.uk

References:

1. Leonhardt, U. Optical conformal mapping. Science 312, 1777-1780 (2006).

2. Pendry, J. B., Schurig, D. and Smith, D. R. Controlling electromagnetic fields. Science 312, 17801782 (2006).

3. Thacker, V. V. et al., DNA origami based assembly of gold nanoparticles dimers for surfacedenhanced Raman scattering. Nat. Commun. 5, 3448 (2014).

4. Kuzyk, A. et al., DNA-based self-assembly of chiral plasmonic nanostructures with tailored optical response. Nature 483, 311-314 (2012).

5. Kuzyk, A. et al., Reconfigurable 3D plasmonic metamolecules. Nat. Mater. XX, XXX-XXX (2014).

6. Rothemund, P. W. K. Folding DNA to create shapes and patterns. Nature 440, 297-302 (2006).

7. Aldaye, F. A., Palmer, A. L. and Sleiman, H. F., Assembling materials with DNA as the guide. Science 321, 1795-1799 (2008).

8. De Luca, A. et al., Reproducible surface-enhanced Raman quantification of biomarkers in multicomponent mixtures. ACS Nano 8, 2575-2583 (2014).

9. Anderson, P. W. More is different. Science 177, 393-396 (1972). 
a)

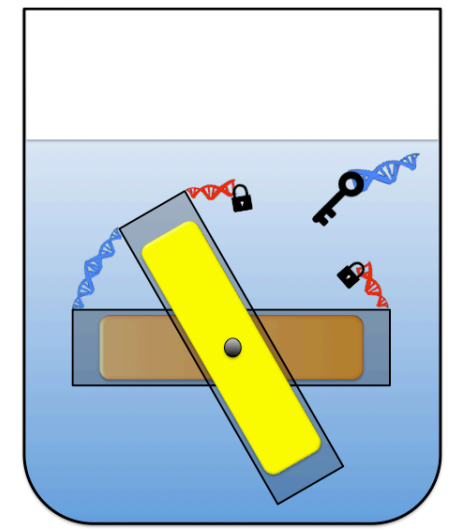

b)

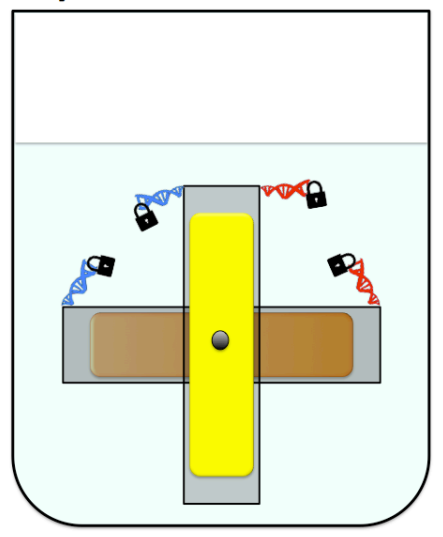

c)

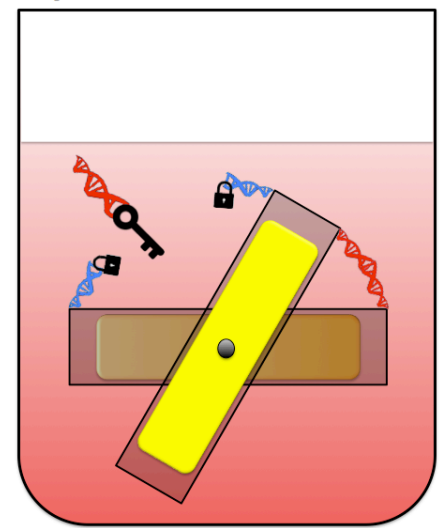

Figure 1 | Sketch of the DNA-driven mechanism for tuning the configuration of assemblies of metal nanorods in solution ${ }^{5}$. The shape and chirality (a, left-handed; b, achiral; c, right-handed) of two gold nanorods (yellow and brown) bound to DNA-origami bundles (grey) and joined at their centre of mass can be altered with two different DNA strands engineered to uncap and connect nearby edges of the bundles. The assemblies are designed to absorb red light with a chirality-dependent efficiency for opposite circular polarizations. 